\title{
A neuropathological study of vascular factors in late-life depression
}

\author{
A J Thomas, I N Ferrier, R N Kalaria, R H Perry, A Brown, J T O’Brien
}

\begin{abstract}
Objectives-Depression is a common psychiatric disorder in late life and it may be associated with vascular disease processes. Although there are clinical and neuroimaging studies lending support to such a "vascular depression" hypothesis there have been no neuropathological studies to directly test this. Postmortem tissue was investigated to determine whether late life depression was associated with atheromatous change in large and medium vessels and microvascular disease in the brain.

Methods-Postmortem tissue wae obtained from 20 patients with a history of at least one episode of DSM-IV major depression and 20 control subjects. Standard procedures were carried out to analyze and quantify Alzheimer type pathology (plaques, tangles, Braak staging) and cortical Lewy bodies. Coronary arteries, cerebral vessels, and aorta were rated for atheromatous disease on a $0-3$ scale and the four neocortical areas were rated for microvascular disease.

Results-The two groups showed no significant differences in age, sex, or postmortem delay. There was a significant increase in atheromatous disease in the depressed group $(p=0.023)$. No differences were found for microvascular disease, either in the brain generally or locally in the frontal lobes. No subject had any significant Alzheimer type or Lewy body pathology.

Conclusions-Neuropathological evidence was found for an excess of atheromatous disease, related to the aortic and cerebral vessels, in late life depression. However, there was no evidence of an increase in microvascular disease. The findings broadly support the vascular depression hypothesis.

(F Neurol Neurosurg Psychiatry 2001;70:83-87)
\end{abstract}

Keywords: depression; pathology; vascular factors

Neuropathology,

Newcastle General

Hospital, Newcastle

upon Tyne, UK

A Brown

Correspondence to: Dr A J Thomas, Wolfson Research Centre, Institute for the Health of the Elderly, Newcastle General Hospital,

Newcastle upon Tyne

NE4 6BE, UK

a.j.thomas@ncl.ac.uk

Received 17 December 1999 and in final form

17 July 2000

Accepted 7 August 2000 ing evidence from both clinical and neuroimaging studies.

Clinical studies have shown strong links in both directions between depression and some vascular disorders. Depression is increased after a myocardial infarction and independently predicts an increase in mortality of three and a half times during the next 6 months. ${ }^{4}$ However, prospective studies of initially healthy people have shown depression also to be an independent risk factor which increases the risk threefold to fourfold of subsequent coronary artery disease and myocardial infarction. ${ }^{45}$ Poststroke depression is a well recognised and common occurrence with high rates of depression reported ranging from 20$65 \% .{ }^{67}$ The Almeda County Study recently demonstrated depressive symptoms to be an independent risk factor for subsequent fatal stroke, increasing fatal stroke by $50 \%$ over 29 years. ${ }^{8}$ Hypertension is associated with a threefold increase in DSM-III major depression ${ }^{9}$ and a large, prospective study has shown depressive symptoms to be a risk factor which doubles the rates of subsequent hypertension. ${ }^{10}$ Finally, depression is increased in dementia, and this is especially the case when it is a vascular dementia. ${ }^{11}$

Magnetic resonance imaging has consistently shown an increase in white matter lesions (WMLs) or signal hyperintensities in depression. ${ }^{12}{ }^{13}$ These are predominantly in the deep white matter ${ }^{14}$ and subcortical grey matter. ${ }^{14}{ }^{16}$ Such lesions are most strongly linked to depression when they involve neuroanatomical sites which underpin frontal-subcortical circuits. ${ }^{17}$ Although WMLs occur in different disorders and arise from a range of pathological processe $^{18}$ deep WMLs may be vascular in origin, representing areas of ischaemia and infarction. ${ }^{19} 20$ They have been linked to hypertension as both a risk factor ${ }^{21}$ and a proposed cause. ${ }^{22}$

In depression no neuropathological studies of WMLs have been carried out to determine whether they are in fact vascular in origin and neither has there been any neuropathological assessment of vascular disease in depression analogous to that carried out in vascular dementia. $^{23}$ We have carried out a study looking at vascular factors in depression to test whether there is evidence of vascular disease associated with late life depression. Our hypotheses were that there would be increased atheroma in the large and medium sized arteries and increased markers of small vessel disease in the brain parenchyma in depressed patients compared to controls. 
Methods

SUBJECTS

Materials from subjects were obtained from the Newcastle brain bank. Full ethical approval has been granted for this postmortem study and consent for postmortem examination obtained from the next of kin.

CLINICAL ASSESSMENT

Depressed patients were included if they were 60 years or older at death and had had at least one well documented episode of DSM-IV major depression. ${ }^{24}$ They were all patients referred to hospital with depression whose diagnosis was made during life by experienced psychiatrists and DSM-IV criteria were applied by case note analysis after death. Subjects were excluded if they had committed suicide by violent means-for example, hanging or carbon monoxide poisoning, or had ever met DSM-IV criteria for dementia, schizophrenia, or other psychotic disorders, a manic or hypomanic episode, or a mood disorder due to a general medical condition. Patients with cognitive impairment during depressive episodes were not excluded provided it was regarded clinically as secondary to the depressed mood and the brain showed no neuropathological evidence of changes consistent with a dementia at postmortem. Control patients met the same criteria except, in addition, they had never had a depressive episode. These were drawn from non-psychiatric patients dying elsewhere in the hospital and case note analysis showed no evidence of any ever having had a depressive episode, although depression earlier in life could have occurred and not been recorded. All depressed patients had undergone extensive assessments including a full history, mental state examination, physical examination, screening blood tests, cognitive tests and, in some cases, CT or MR scans. We obtained information from the notes on whether patients had a history of angina, diabetes mellitus, atrial fibrillation, cardiac failure, or hypertension (sufficient to warrant antihypertensive treatment) and on whether they had ever smoked. We used the postmortem assessment of the heart to determine which patients had had a myocardial infarction. Finally potential patients were excluded if there was clinical or neuropathological evidence they had died from a hypoxic episode.

\section{NEUROPATHOLOGICAL ASSESSMENT}

All patients received a full postmortem examination (except one whose body was taken by the undertakers after the brain was removed) and the delay to postmortem was recorded. The brains were dissected in a standard manner. Both the left (sliced fresh and snap frozen) and right (formalin fixed) hemispheres were inspected for infarcts and ischaemia and their presence recorded. Tissue blocks from the right hemisphere were taken from the frontal, temporal, parietal, and occipital cortices, the hippocampus, the cingulate, the basal ganglia; the cerebellum, the upper and lower midbrain, the pons and the medulla. Sections of $5 \mu \mathrm{m}$ or $20 \mu \mathrm{m}$ were cut and sections were prepared on glass slides. They were stained with haematoxylin and eosin, luxol fast blue and/or Loyez, cressyl fast violet, methanamine silver and/or von Braunmuhl, Palmgren and/or tau-2, and ubiquitin. They were then examined by an experienced neuropathologist (RHP) and patients were excluded if they met neuropathological criteria for Alzheimer's disease or other known causes of dementia or had evidence of any other neurological disorder.

As we wanted to examine a pure (nondemented) group of depressive patients and controls specific histopathological analysis was carried out to quantify the neuritic plaques and neurofibrillary tangles in the four neocortical lobes. ${ }^{25}$ Plaques were counted in five fields (which included at least one from the gyral crest, at least one from the sulcul depths, and included all cortical layers) from each neocortical area (frontal, temporal, parietal, occipital). A mean score was obtained and converted to numbers $/ \mathrm{mm}^{2}$. Neocortical tangles were also counted in five fields in a similar manner except that each count was the mean across all the cortical layers. Alzheimer type pathology was also staged according to the procedure of Braak and Braak, ${ }^{26}$ which involves assessing numbers of neurofibrillary tangles and neuropil threads in different parts of the hippocampus (stages 1-4) and neocortex (stages 5 and 6). Lewy bodies were counted as absolute numbers in an area measured using a Kontron Videoplan Image analysis computer and scores were converted to numbers $/ \mathrm{cm}^{2}$. The sections for this were taken from the various brain areas described in the concensus guidelines for dementia with Lewy bodies. ${ }^{27}$

ASSESSMENT OF LARGE AND MEDIUM SIZED ARTERIES

The severity of atheroma was rated by a neuropathologist who was unaware of the nature of the study or its hypotheses and therefore who were cases and controls. The most severe atheromatous disease in a vessel was rated in all the main coronary arteries (left anterior descending, circumflex, and right coronary artery), the arteries of the carotid and vertebrobasilar systems bilaterally, and aorta semiquantitatively on a 1-3 scale: $1=$ mild: atheroma only mild and patchy; $2=$ moderate: more extensive atheroma with occlusion of lumen less than 50\%; 3=severe: atheroma widespread with greater than 50\% luminal occlusion in at least one vessel. The aortic assessment was based on the percentage and severity of intimal area affected. An overall atheroma score was obtained for each patient by adding together the scores from each of these.

\section{ASSESSMENT OF SMALL ARTERIES AND}

\section{ARTERIOLES}

For assessing microvascular disease the sections stained with haematoxylin and eosin, luxol fast blue and/or Loyez, and cressyl fast violet from the frontal, temporal, pareital and occipital cortices were used. In addition large full face coronal blocks were taken from the frontal cortices to include all the deep white 
Table 1 Demographic and microscopic features of the depressed and control groups

\begin{tabular}{|c|c|c|c|c|c|}
\hline & $\begin{array}{l}\text { Depressed group } \\
n=20\end{array}$ & $\begin{array}{l}\text { Control group } \\
n=20\end{array}$ & $\begin{array}{l}\text { Test } \\
\text { statistic }\end{array}$ & $p$ Value & $95 \% C I$ \\
\hline Age $(y)^{\star}$ & $75.0(7.37)$ & $74.2(7.46)$ & 0.30 & 0.77 & -5.45 to 4.05 \\
\hline $\operatorname{Sex}(M / F)$ & $7 / 13$ & $7 / 13$ & & 1.00 & \\
\hline Age of onset of depression $(y)^{\star}$ & $63.8(14.83)$ & NA & NA & NA & \\
\hline Postmortem delay $(\mathrm{h})^{\star}$ & $34.55(22.72)$ & $28.0(16.18)$ & 1.05 & 0.30 & -19.18 to 6.08 \\
\hline Plaque count $(/ \mathrm{mm})^{\star}$ & $1.5(1.74)$ & $1.36(2.96)$ & 134 & 0.07 & \\
\hline Tangle count $(/ \mathrm{mm})^{\star}$ & $0.058(0.20)$ & $0.006(0.014)$ & 184 & 0.68 & \\
\hline Braak stage & 1 & 1 & 6.25 & 0.18 & \\
\hline Lewy body count $\left(\mathrm{cm}^{2}\right)^{\star}$ & $0(0)$ & $0(0)$ & NA & 1.00 & \\
\hline Total microvasculart & 4.0 & 4.5 & 7.24 & 0.56 & \\
\hline Frontal microvasculart & 1.0 & 1.0 & 0.70 & 0.79 & \\
\hline
\end{tabular}

*Values are mean (SD) and comparisons are made using unpaired $t$ tests except for plaque and tangle scores which are Mann-Whitney tests.

tValues are medians and comparisons are made using Fisher-Freeman-Halton tests.

matter in this area. Sections of $10 \mu \mathrm{m}$ or $20 \mu \mathrm{m}$ were prepared on large ( 3 inch $\times 2$ inch) slides and stained with haematoxylin and eosin, luxol fast blue and/or Loyez, and cresyl fast violet. Microvascular disease was then scored blind to diagnosis by two raters (RHP and AJT) in each neocortical area in accordance with a scale previously used in vascular dementia. ${ }^{23}$ Briefly, this is a $0-3$ scale in which $0=$ normal; $1=$ dilatation of perivascular spaces or hyaline thickening or a few perivascular macrophages; $2=$ dilatation of perivascular spaces or hyaline thickening plus mild to moderate perivascular pallor of myelin staining or loosening of the nerve fibres or nerve cells with gliosis; $3=$ Binswanger's disease (2 plus more widespread myelin pallor, nerve fibre or nerve cell disruption and gliosis). An overall microvascular score was calculated for each patient by adding together the scores for each neocortical area. A separate score was obtained by assessment of the frontal deep white matter.

\section{STATISTICS}

The two groups were compared using SPSS software (version 9.0) to carry out unpaired Student's $t$ tests or Mann-Whitney $U$ tests, as appropriate, on the quantitative data (age, postmortem interval, plaques, tangles, Lewy bodies). Fisher-Freeman-Halton tests (StatXact version 4) were used to compare the groups on the categorical data (Braak, atheroma, microvascular) and comparisons of the groups on clinical cardiovascular risk factors were carried out using the Fisher's exact test (StatXact version 4) because low cell frequencies meant the $\chi^{2}$ test was inappropriate for this categorical data.

\section{Results}

Twenty patients with depression and twenty controls were obtained and their characteristics are shown in table 1 . There were no significant differences in age, sex or postmortem delay between the two groups $(p>0.17)$. The causes of death (controls/depressed) were: cardiac 8/7; pneumonia $3 / 7$; other respiratory $2 / 2$; carcinoma $4 / 1$; suicide $0 / 2$ (both overdose of alcohol and tranquillisers); other 3 (haematemesis, mesenteric infarction, liver failure)/1(renal failure).

The figure shows the distribution of atheroma scores. These were significantly higher in the depressed group $(p=0.023$ (Fisher statistic 11.2)). A breakdown of the atheroma scores showed the significant difference to be due to a trend towards increased atheroma in both the cerebral $(\mathrm{p}=0.096$; Fisher statistic 3.9) and aortic ( $p=0.060$; Fisher statistic 5.71) vessels rather than in the coronary arteries ( $p=1.00$; Fisher statistic 0.19 ;).

The pathological features rated microscopically are shown in table 1 . There were no Lewy bodies detected in either group and both groups also had very low levels of Alzheimer type pathology. ${ }^{25}$ A comparison showed no significant differences between the two groups on plaque count $(\mathrm{p}=0.07 ; U=134)$, tangle count $(p=0.68 ; U=184$,$) or Braak stage (p=0.18$; Fisher statistic 6.25). The two groups were also very similar in their microvascular scores both generally over the four neocortical areas $(p=0.56$; Fisher statistic 7.24$)$ and specifically in the deep white matter of the frontal lobe $(\mathrm{p}=0.79$; Fisher statistic 0.70).

Table 2 shows the data and comparisons for the two groups on clinical cardiovascular risk factors. The data on myocardial infarction were complete (the one patient whose body was removed was rated from his history) and complete data were obtained on all the depressed patients except for the smoking data. No clinical information on cardiovascular risk could be obtained at all on eight control subjects and on two subjects information was incomplete. The available data showed no significant differences between the groups and overall table 2 shows a slight excess of cardiovascular risk in the control group.

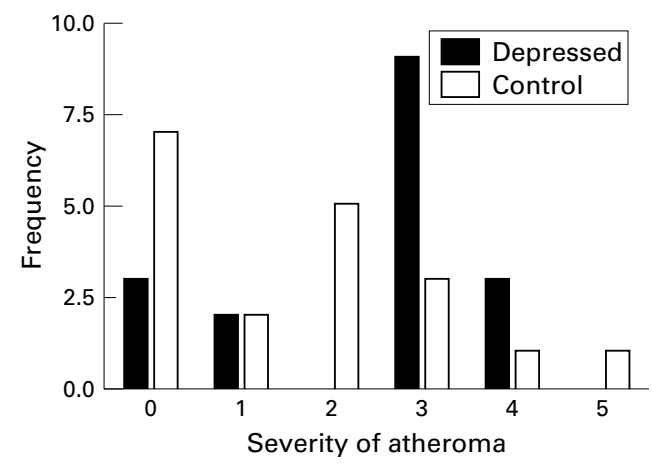

Frequency of different severities of atheroma in 20 depressed patients and 20 control subjects. The

Fisher-Freeman-Halton test shows a significant excess of atheroma in the depressed group ( $p=0.023$; Fisher statistic 11.2). 
Table 2 Clinical vascular risk factors

\begin{tabular}{lllll}
\hline Risk factor & Depressed $n(\%)$ & Control n(\%) & Fisher statistic & p Value \\
\hline Myocardial infarction & $3(15)$ & $5(25)$ & 0.63 & 0.70 \\
Angina & $3(15)$ & $4(40)$ & 2.27 & 0.18 \\
Hypertension & $7(35)$ & $4(33)$ & 0.062 & 1.00 \\
Diabetes mellitus & $0(0)$ & $2(20)$ & 3.58 & 0.10 \\
Atrial fibrillation & $3(15)$ & $1(10)$ & 0.18 & 1.00 \\
Cardiac failure & $7(35)$ & $10(71)$ & 4.25 & 0.08 \\
Smoking & $7(64)$ & $7(78)$ & 0.49 & 0.64 \\
\hline
\end{tabular}

${ }^{\star}$ History of risk factor except for myocardial infarction, which was determined by postmortem examination.

\section{Discussion}

Our two hypotheses were that there would be an increase in atheroma and in microvascular disease in the depressed group. The first was supported as we found a significant increase in atheroma overall $(\mathrm{p}=0.023)$, with this being due mainly to the difference in the cerebral arteries $(\mathrm{p}=0.096)$ and aorta $(\mathrm{p}=0.060)$, but the second was not because we found no evidence for increased microvascular disease either generally in the four neocortical lobes or specifically within the deep white matter of the frontal lobe.

The hypothesis of "vascular depression" ${ }^{3}$ is important to test because white matter lesions, which are probably vascular in origin, ${ }^{192}$ predict both initial treatment resistance ${ }^{1728}$ and poor long term outcome..$^{29}$ Consequently they may be markers for a potentially treatable and preventable vascular variant of depression. ${ }^{31}$ Our finding of increased atheroma is consistent with the evidence from studies of poststroke depression of the importance of large and medium sized vessel disease in depression. ${ }^{6}$ There is also evidence that the relation applies in both directions because depression has recently been shown to be a risk factor for fatal stroke. ${ }^{8}$ Similarly, the increase in atheroma in the depressed group fits with the evidence, from both cross sectional and cohort studies, of an increased rate of myocardial infarction both before ${ }^{45}$ and after ${ }^{4}$ the diagnosis of depression. The lack of difference in the coronary arteries themselves in our groups may be due to a selection bias in which depression earlier in life is associated with coronary artery disease, which leads to early death for such patients. Our depressed group was both elderly and had a late onset of first episode of depression (mean 63.8 years) and a pattern of atheroma which differentially affects other arteries, especially the cerebral arteries, may be associated with late-life depression. The depressed group was found to be comparable with the control group on clinical cardiovascular risk factors and so the findings are not due to an imbalance in cardiovascular risk in the depressed group. Our finding of excess atheroma in this group of late-life depressive patients potentially extends the relevance of atheromatous disease in large and medium sized vessels to depression more generally in elderly people.

The absence of evidence for a relation of depression with microvascular disease is surprising given the links between depression and hypertension, ${ }^{9}$ and deep white matter disease. ${ }^{14}{ }^{15}$ The second seems to be vascular ${ }^{19} 20$ and specifically microvascular ${ }^{32}$ in origin. It may be that microvascular disease is not itself linked to depression (at least in late life) but there are other explanations for our results. It is possible that there is a subgroup of depressive patients with microvascular disease although we did not find direct evidence for this in this series. The semiquantitative method used is designed for studies of vascular dementia where more obvious pathology is present and the numbers in our groups are also small. Therefore we may have failed to detect real, important, but more subtle differences between the groups. Again more patients in each group would confer a greater power to detect any differences. Two groups of 20 gives $80 \%$ power to detect a difference of about 1 SD and thus we would expect to detect large differences but smaller ones could have been missed. A further limitation in our study is that it was a retrospective case note study and so, for example, we cannot be absolutely sure that a control case had not had depression earlier in life, although controls were only included if hospital records indicated an absence of psychiatric history. Although a failure to detect smaller, real differences in small vessel disease may have occurred the absence of evidence for more widespead small vessel disease may also point to the importance of focusing on specific neural circuitry - for example, frontal-subcortical circuitry - and looking at the impact of white matter disease on this. Whereas we looked at the deep white matter of the frontal lobes for microvascular disease it is possible that a more detailed and complex analysis focusing on the frontal-subcortical circuits may be fruitful because these are the particular sites of interest ${ }^{33}$ in depression and have been proposed as the likely sites of vascular disease. ${ }^{2}$

Both our depressed and control patients had low levels of Alzheimer type pathology and no Lewy body pathology; this is comparable with the levels found in a previous study of a large group of normal controls assessed in the same way. They were also much lower than the levels found in patients who had Alzheimer's disease or dementia with Lewy bodies. ${ }^{25}$ Thus our findings cannot necessarily be extended to late life depressive patients with evidence of a comorbid dementia.

Our finding of increased atheroma in the large and medium sized vessels in late-life depression merits further study. If confirmed it will indicate that people with depression later in life should be screened for atheromatous disease as a factor perpetuating their depression as well as predisposing them to other vascular disorders. This is of great importance as depression is already the fourth leading cause of disability world wide ${ }^{34}$ and is predicted to become the second by $2020 .^{35}$

We thank the Stanley Foundation and the Medical Research Council for financial support for this project.

1 Beekman ATF, Copeland JRM, Prince MJ. Review of community prevalence of depression in later life. Br $\mathcal{F}$ Psychiamunity prevalence of dep

2 Alexopoulos GS, Meyers BS, Young RC, et al. Vascular depression hypothesis. Arch Gen Psychiatry 1997;54:915- 
3 Krishnan KR, Hays JC, Blazer DG. MRI-defined vascular depression. Am f Psychiatry 1997;154:497-501.

Glassman AH, Shapiro PA. Depression and the course of coronary artery disease Am f Psychiatry 1998;155:4-11.

5 Ford DE, Mead LA, Chang PP, et al. Depression is a risk factor for coronary artery disease in men: the precursors study. Arch Intern Med 1998;158:1422-6.

6 Gordon WA, Hibbard MR. Poststroke depression: an examination of the literature. Arch Phys Med Rehabil 1997;78 658-63.

7 Pohjasvaara T, Leppavuori A, Siira I, et al. Frequency and clinical determinants of poststroke depression. Stroke 1998;29:2311-7.

8 Everson SA, Roberts RE, Goldberg DE, et al. Depressive symptoms and increased risk of stroke mortality over a 29 year period. Arch Intern Med 1998;158:1133-8.

9 Rabkin JG, Charles E, Kass F. Hypertension and DSM-III depression in psychiatric outpatients. Am $\mathcal{F}$ Psychiatry depression in psych:1072-4.

10 Jonas BS, Franks P, Ingram DD. Are symptoms of anxiety and depression risk factors for hypertension? Longitudinal andence from the national health and nutrition examinaevidence from the national health and nutrition examination survey I

11 Ballard CG, Patel A, Solis M, et al. A 1 year follow up study of depression in dementia sufferers. $\mathrm{Br} f$ Psychiatry 1996;168:287-91.

12 O'Brien JT, Ames D, Schwietzer I. White matter changes in depression and Alzheimer's disease: a review of magnetic resonance imaging studies. Int f Geriatr Psychiatry 1996;11 681-94.

13 Videbech P. MRI findings in patients with affective disorder: a meta-analysis. Acta Psychiatr Scand 1997;96:157-68.

14 Rabins PV, Pearlson GD, Aylward E, et al. Cortical magnetic resonance imaging changes in elderly inpatients with major depression. Am f Psychiatry 1991;148:617-20.

15 O'Brien J, Desmond P, Ames D, et al. A magnetic resonance imaging study of white matter lesions in depression and imaging study of white matter lesions in depression

16 Greenwald BS, Kramer-Ginsberg E, Krishnan RR, et al. MRI signal hyperintensities in geriatric depression. Am $\mathcal{F}$ MRI signal hyperintensities

17 Greenwald BS, Kramer-Ginsberg E, Krishnan KR, et al. Neuroanatomic localization of magnetic resonance imaging signal hyperintensities in geriatric depression. Strok 1998;29:613-7.

18 Everall IP, Chong WK, Wilkinson ID, et al. Correlation of MRI and neuropathology in AIDS. F Neurol Neurosurg Psychiatry 1997;62:92-5.

19 Awad IA, Johnson PC, Spetzler RF, et al. Incidental subcortical lesions identified on magnetic resonance imaging in the elderly. II. Postmortem pathological correlations. Stroke 1986;17:1090-7.
20 Fazekas F, Kleinert R, Offenbacher H, et al. Pathologic correlates of incidental MRI white matter signal hyperintensirelates of incidental MRI white

21 Fujikawa T, Yamawaki S, Touhouda Y. Background factors and clinical symptoms of major depression with silent cerebral infarction. Stroke 1994;25:798-801.

22 Skoog I. A review on blood pressure and ischaemic white matter lesions. Dement Geriatr Cogn Disord 1998;9(suppl 1):13-9.

23 Esiri MM, Wilcock GK, Morris JH. Neuropathological assessment of the lesions of significance in vascular dementia. F Neurol Neurosurg Psychiatry 1997;63:749-53.

24 American Psychiatric Association. Diagnostic and statistical manual of mental disorders. 4th edition. Washington, DC: APA, 1994

25 Perry R, Jaros E, Irving D, et al. What is the neuropathological basis of dementia associated with Lewy bodies? In: RH Perry, IG McKeith, EK Perry, eds. Dementia with Lewy bodies. Cambridge: Cambridge University Press, 1996:21223.

26 Braak H, Braak E. Neuropathological stageing of Alzheimer related changes. Acta Neuropathol (Berl) 1991;82:239-59.

27 McKeith IG, Galasko D, Kosaka K, et al. Consensus guidelines for the clinical and pathologic diagnosis of dementia with Lewy bodies (DLB): report of the consortium on DLB international workshop. Neurology 1996;47:1113-24.

28 Simpson S, Baldwin RC, Jackson A, et al. Is subcortical disease associated with a poor response to antidepressants? Neurological, neuropsychological and neuroradiological findings in late-life depression. Psychol Med 1998;28:101526.

29 Simpson SW, Jackson A, Baldwin RC, et al. 1997 IPA/Bayer research awards in psychogeriatrics. Subcortical hyperintensities in late-life depression: acute response to treatment and neuropsychological impairment. Int Psychogeriatr 1997;9:257-75.

30 O'Brien J, Ames D, Chiu E, et al. Severe deep white matter lesions and outcome in elderly patients with major depressive disorder: follow up study. BMF 1998;317:982-4.

31 Hickie I, Scott E. Late-onset depressive disorders: a preventable variant of cerebrovascular disease? Psychol Med 1998;28:1007-13.

32 Mantyla R, Aronen H, Salonen O, et al. Magnetic resonance maging white matter hyperintensities and mechanism of ischemic stroke. Stroke 1999;30:2053-8.

33 Cummings JL. Frontal-subcortical circuits and human behavior. Arch Neurol 1993;50:873-80.

34 Murray CJ, Lopez AD. Global mortality, disability, and the contribution of risk factors: global burden of disease study. Lancet 1997;349:1436-42.

35 Murray CJ, Lopez AD. Alternative projections of mortality and disability by cause 1990-2020: global burden of disease study. Lancet 1997;349:1498-504. 